\title{
The breaking of the Equivalence Principle in theories with varying $\alpha$
}

\section{Lucila Kraiselburd ${ }^{1,2}$ and Héctor Vucetich ${ }^{1}$}

${ }^{1}$ Facultad de Ciencias Astronómicas y Geofísicas, Universidad Nacional de La Plata, Argentina

email: vucetich@fcaglp.fcaglp.unlp.edu.ar

${ }^{2}$ CONICET, Argentina

email: lulikrai@gmail.com

\begin{abstract}
The Standard Model and General Relativity provide a good description of phenomena at low energy. These theories, which agree very well with the experiment, contain a set of parameters called "fundamental constants", that are assumed invariant under changes in location and reference system. However, their possible variation has been studied since Dirac made the large numbers hypothesis (LNH). Moreover, unified field theory and extra dimensions theories such as Kaluza-Klein or Superstring theories, state not only the variation of these constants, but also the simultaneity of the variations.

The Eötvös effect is one of the most sensitive indicators of changes in fundamental constants. Bekenstein (2002) showed that in his theory, using a classical static particle model of matter, there is no Eötvös effect and therefore met the Universality of Free Fall and the Principle of Equivalence.

We present different results than those obtained by Bekenstein, Kraiselburd, Vucetich (2009). Modifying his theory, taking more realistic models of matter and using the model $T H \epsilon \mu$ techniques (Ligtman-Lee (1975) and Haugan (1979), not used before to analyze this model), very small but measurable effects have been found.
\end{abstract}

\section{References}

L. Kraiselburd \& H. Vucetich. "Violation of the Weak Equivalence Principle in Bekenstein's theory". arXiv:0902.4146, 2009.

Jacob D. Bekenstein. "Fine-structure constant variability, equivalence principle and cosmology". Phys. Rev., D66:123514, 2002.

M. P. Haugan. "Energy conservation and the principle of equivalence". Ann. Phys., 118:156, 1979.

A. P. Lightman \& D. L. Lee. "Restricted proof that the Weak Equivalence Principle implies the Einstein Equivalence Principle". Phys. Rev. D, 8:364, 1973. 